\title{
Symbiotic gap and semigap solitons in Bose-Einstein condensates
}

\author{
Sadhan K. Adhikari ${ }^{1}$ and Boris A. Malomed ${ }^{2}$ \\ ${ }^{1}$ Instituto de Física Teórica, UNESP-São Paulo State University, 01.405-900 São Paulo, São Paulo, Brazil \\ ${ }^{2}$ Department of Physical Electronics, School of Electrical Engineering, Tel Aviv University, Tel Aviv 69978, Israel
}

(Received 11 September 2007; revised manuscript received 7 November 2007; published 5 February 2008)

\begin{abstract}
Using the variational approximation and numerical simulations, we study one-dimensional gap solitons in a binary Bose-Einstein condensate trapped in an optical-lattice potential. We consider the case of interspecies repulsion, while the intraspecies interaction may be either repulsive or attractive. Several types of gap solitons are found: symmetric or asymmetric; unsplit or split, if centers of the components coincide or separate; intragap (with both chemical potentials falling into a single band gap) or intergap, otherwise. In the case of the intraspecies attraction, a smooth transition takes place between solitons in the semi-infinite gap, those in the first finite band gap, and semigap solitons (with one component in a band gap and the other in the semi-infinite gap).
\end{abstract}

DOI: 10.1103/PhysRevA.77.023607

PACS number(s): 03.75.Ss, 03.75.Lm, 05.45.Yv

\section{INTRODUCTION}

One of the milestones in studies of Bose-Einstein condensates (BECs) was the creation of bright solitons in ${ }^{7} \mathrm{Li}$ and ${ }^{85} \mathrm{Rb}$ in "cigar-shaped" traps [1], with the atomic scattering length made negative (which corresponds to the attraction between atoms) by means of the Feshbach-resonance (FR) technique [2]. Normally, BEC features repulsion among atoms. In that case, it was predicted that an optical-lattice (OL) potential may support gap solitons (GSs) [3], whose chemical potential falls in finite band gaps of the OL-induced spectrum. Although GSs, unlike ordinary solitons in selfattractive BEC, cannot realize the ground state of the condensate, it was demonstrated that they may easily be stable against small perturbations [4]. A GS in ${ }^{87} \mathrm{Rb}$ was experimentally created in a cigar-shaped trap combined with an OL potential, pushing the BEC into the appropriate band gap by acceleration [5]. Other possibilities for the creation of GSs are offered by phase imprinting [6], or squeezing the system into a small region by a tight longitudinal parabolic trap, which is subsequently relaxed [7].

BEC mixtures of two hyperfine states of the same atom are also available to the experiment [8]. The sign and strength of the interspecies interaction may also be controlled by means of the FR [9], hence one may consider a binary condensate with intraspecies repulsion combined with attraction between the species. It was proposed to use this setting for the creation of symbiotic solitons [10], in which the attraction overcomes the intrinsic repulsion.

In this work, we aim to study compact (tightly bound [11]) symbiotic gap solitons in a binary BEC, which are trapped, essentially, in a single cell of the underlying OL potential. Unlike the situation dealt with in Refs. [10], we consider the case of interspecies repulsion, while the intraspecies interactions may be repulsive or attractive. In Ref. [11] it was already demonstrated that the addition of intraspecies repulsion expands the stability region of symbiotic GSs supported primarily by the interspecies repulsion. The case of attraction between two self-repulsive species was recently considered in Ref. [12], where it was shown that the attraction leads to a counterintuitive result—splitting be- tween GSs formed in each species. This effect can be explained by a negative effective mass, which is a characteristic feature of the GS [3]. Indeed, considering the interaction of two GSs belonging to different species, one may expect that the interplay of the attractive interaction with the negative mass will split the GS pair.

Using variational [13] and numerical methods, we here construct families of stable GSs of two kinds: unsplit (fully overlapping) and split (separated). The splitting border is predicted by the variational approximation (VA) in an almost exact form. In terms of chemical potentials of the two components, the solitons may be of intragap and intergap types [11], with the two components sitting, respectively, in the same gap or different gaps. In particular, the states with one component residing in the semi-infinite gap (which is possible in the case of intraspecies attraction) will be called semigap solitons.

The paper is organized as follows. The formulation of the system and analytical results, obtained by the variational method [13], are given in Sec. II. Numerical findings are reported in Sec. III, including maps of GS families in appropriate parameter planes. Section IV summarizes the work.

\section{ANALYTICAL CONSIDERATIONS}

We consider a binary BEC loaded into a cigar-shaped trap combined with an OL potential acting in the axial direction. Starting with the system of coupled 3D Gross-Pitaevskii equations (GPEs) for wave functions of the two components $\phi_{1}$ and $\phi_{2}$, one can reduce them to one-dimensional (1D) equations [14]. In the scaled form, they are [12]

$$
\begin{aligned}
i\left(\phi_{1,2}\right)_{t}= & -(1 / 2)\left(\phi_{1,2}\right)_{x x}+g\left|\phi_{1,2}\right|^{2} \phi_{1,2}+g_{12}\left|\phi_{2,1}\right|^{2} \phi_{1,2} \\
& -V_{0} \cos (2 x) \phi_{1,2},
\end{aligned}
$$

where the OL period is fixed to be $\pi$, and the wave functions are normalized to numbers of atoms in the two species, $\int_{-\infty}^{+\infty}\left|\phi_{1,2}(x)\right|^{2} d x=N_{1,2}$. In Eq. (1), time, the OL strength, and nonlinearity coefficients are related to their counterparts measured in physical units as follows: $t \equiv(\pi / L)^{2}(\hbar / m) t_{\text {phys }}$, $V_{0} \equiv(L / \pi \hbar)^{2} m\left(V_{0}\right)_{\text {phys }}, \quad\left\{g, g_{12}\right\} \equiv\left(2 L m \omega_{\perp} / \pi \hbar\right)\left\{a, a_{12}\right\}$, 
where $m$ is the atomic mass, $L$ is the OL period, $a$ and $a_{12}$ are scattering lengths accounting for collisions between atoms belonging to the same or different species, and $\omega_{\perp}$ is the transverse-confinement frequency. As said above, we assume repulsive interspecies interactions, with $g_{12}>0$, while the intraspecies nonlinearity may be both repulsive $(g>0)$ and attractive $(g<0)$.

While the model assumes equal intraspecies scattering lengths, they are, in general, different for two hyperfine states [8]. Therefore, using a FR, one cannot modify both intraspecies nonlinearities to keep exactly equal values of coefficient $g$ in equations for both components [cf. Eq. (1)], running from negative to positive values (hence, strictly speaking, different cases considered in this work cannot be realized in a single mixture, but should be rather considered as a collection of situations occurring in different mixtures). However, we will consider asymmetric configurations, with $N_{1} \neq N_{2}$, which give rise to a much stronger difference in the effective interaction strengths in the two components than a small difference in their intrinsic scattering lengths.

Stationary solutions to Eqs. (1) are looked for in the usual form, $\phi_{1,2}(x, t)=\exp \left(-i \mu_{1,2} t\right) u_{1,2}(x)$, with chemical potentials $\mu_{1,2}$ and functions $u_{1,2}(x)$ obeying

$$
\mu_{1,2} u_{1,2}+u_{1,2}^{\prime \prime} / 2-g u_{1,2}^{3}-g_{12} u_{2,1}^{2} u_{1,2}+V_{0} \cos (2 x) u_{1,2}=0,
$$

with $\int_{-\infty}^{+\infty} u_{1,2}^{2}(x) d x=N_{1,2}$. In the GS solutions constructed below, $\mu_{1}$ and $\mu_{2}$ belong to the first two finite band gaps and/or the semi-infinite gap in the spectrum induced by potential $-V_{0} \cos (2 x)$.

Variational approximation for unsplit solitons. Equation (2) can be derived from Lagrangian

$$
\begin{aligned}
L= & \int_{-\infty}^{+\infty}\left[\mu_{1} u_{1}^{2}+\mu_{2} u_{2}^{2}-\frac{1}{2}\left(\left(u_{1}^{\prime}\right)^{2}+\frac{1}{2}\left(u_{2}^{\prime}\right)^{2}\right)+V_{0} \cos (2 x)\right. \\
& \left.\times\left(u_{1}^{2}+u_{2}^{2}\right)-\frac{1}{2} g\left(u_{1}^{4}+u_{2}^{4}\right)-g_{12} u_{1}^{2} u_{2}^{2}\right] d x-\mu_{1} N_{1}-\mu_{2} N_{2} .
\end{aligned}
$$

To predict solitons with a compact symmetric profile, which corresponds to numerical results displayed below, we adopt the Gaussian ansatz [13]

$$
u_{1,2}^{\text {(unsplit) }}(x)=\pi^{-1 / 4} \sqrt{\frac{N_{1,2} \aleph_{1,2}}{w_{1,2}}} \exp \left(-\frac{x^{2}}{2 w_{1,2}^{2}}\right),
$$

where variational parameters are widths $w_{1,2}$, reduced norms $\aleph_{1,2}$, and $\mu_{1,2}$. The substitution of the ansatz in Eq. (3) yields an effective Lagrangian, $L=L\left(\aleph_{1,2}, w_{1,2}, \mu_{1,2}\right)$. Then, the first pair of the variational equations, $\partial L / \partial \mu_{1,2}=0$, gives $\aleph_{1,2}=1$, which is substituted below, after performing the variation with respect to $\aleph_{1,2}$. Thus, the remaining equations, $\partial L / \partial w_{1,2}=\partial L / \partial \aleph_{1,2}=0$, take the form

$$
1+\frac{g N_{1,2} w_{1,2}}{\sqrt{2 \pi}}+\frac{2 g_{12} N_{2,1} w_{1,2}^{4}}{\sqrt{\pi}\left(w_{1}^{2}+w_{2}^{2}\right)^{3 / 2}}=4 V_{0} w_{1,2}^{4} e^{-w_{1,2}^{2}},
$$

$$
\mu_{1,2}=\frac{1}{4 w_{1,2}^{2}}+\frac{g N_{1,2}}{\sqrt{2 \pi} w_{1,2}}+\frac{g_{12} N_{2,1}}{\sqrt{\pi\left(w_{1}^{2}+w_{2}^{2}\right)}}-V_{0} e^{-w_{1,2}^{2}}
$$

Using Eqs. (5) and (6) we can predict borders between intragap and intergap soliton families of different types. To this end, we take $\mu_{1,2}$ from Eqs. (6) and, referring to the spectrum of the linearized equation (1), identify curves in plane $\left(N_{1}, N_{2}\right)$ which correspond to boundaries between different gaps in the two components.

Variational approximation for split solitons. Twocomponent solitons different from those considered above feature splitting between the two components. An issue of obvious interest is to predict the splitting threshold by means of the VA, for the symmetric case, with $N_{1}=N_{2} \equiv N$. For this purpose, we use the following ansatz:

$$
\begin{aligned}
u_{1,2}^{\text {(split })}(x)= & \pi^{-1 / 4} \sqrt{\frac{N}{w}}\left[1 \pm b x+\frac{C}{4} w^{2} b^{2}-\frac{1}{2}(1+C) b^{2} x^{2}\right] \\
& \times \exp \left(-\frac{x^{2}}{2 w^{2}}\right),
\end{aligned}
$$

with infinitesimal splitting parameter $b$, the objective being to find a point at which a solution with $b \neq 0$ emerges. At small $b$, the two components of expression (7) feature maxima shifted to $x= \pm b / a+O\left(b^{2}\right)$, and up to order $b^{2}$, it satisfies the normalization conditions, $\int_{-\infty}^{\infty} u_{1,2}^{2}(x) d x=N$. Unlike $b$, constant $C$, to be defined below, is not a variational parameter.

The substitution of ansatz (7) in Lagrangian (3) yields, at orders $b^{0}$ and $b^{2}$,

$$
\begin{aligned}
L= & -\frac{N}{2 w^{2}}+2 V_{0} e^{-w^{2}}-\frac{g+g_{12}}{\sqrt{2 \pi} w} N^{2}-b^{2} N\left[1+\frac{C}{2}\right. \\
& \left.-2 C V_{0} w^{4} e^{-w^{2}}+\frac{g(C+2)+(C-2) g_{12}}{2 \sqrt{2 \pi}} w N\right] .
\end{aligned}
$$

At order $b^{0}$ (i.e., for the unsplit soliton), variational equation $\partial L / \partial w=0$ reduces to Eq. (5) with $N_{1}=N_{2} \equiv N$ and $w_{1}=w_{2}$ $\equiv w$ as follows:

$$
1+\frac{\left(g+g_{12}\right) N w}{\sqrt{2 \pi}}=4 V_{0} w^{4} e^{-w^{2}} .
$$

At order $b^{2}$, equation $\partial L / \partial\left(b^{2}\right)=0$ yields the splitting condition,

$$
\frac{C+2}{4}\left(1+\frac{g N w}{\sqrt{2 \pi}}\right)+\frac{C-2}{4} \frac{g_{12} N w}{\sqrt{2 \pi}}-C V_{0} w^{4} e^{-w^{2}}=0 .
$$

Obviously, the splitting should not occur if $g_{12}=0$, i.e., Eq. (10) must only yield the trivial solution $w=0$ in this case. This condition selects the value of $C$ which was arbitrary hitherto: $C=-2$, hence Eq. (10) takes the form $\mathrm{Ng}_{12}$ $=2 \sqrt{2 \pi} V_{0} w^{3} e^{-w^{2}}$. Combining this with Eq. (9), we obtain 

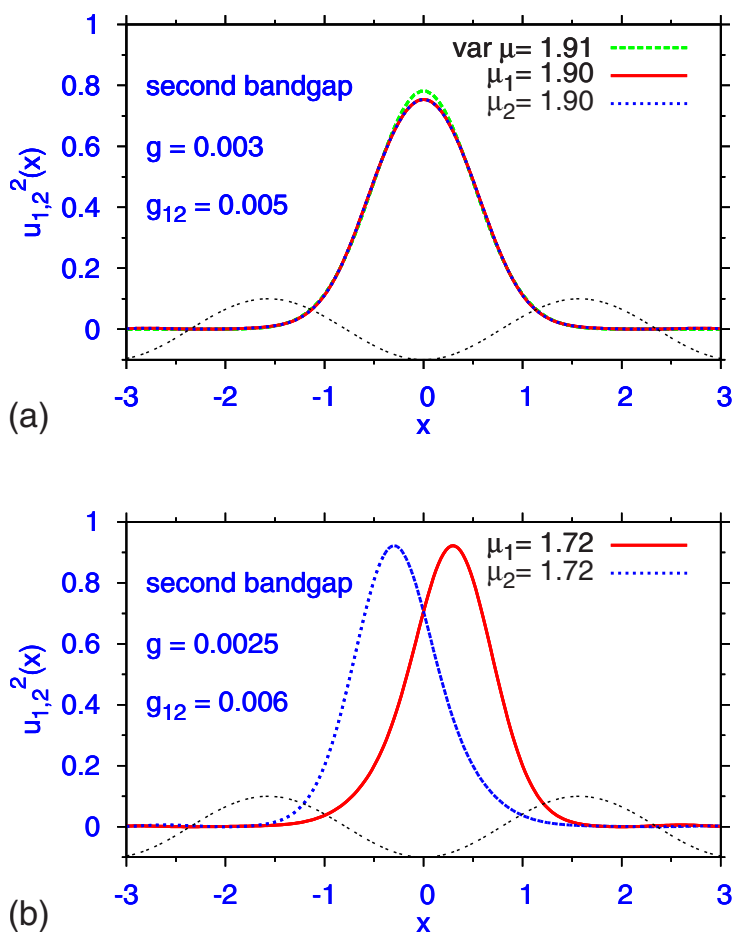

FIG. 1. (Color online) Examples of unsplit and split symmetric solitons, with $N_{1}=N_{2}=1000$, trapped in potential $-V_{0} \cos (2 x)$. Here and in all other figures, $V_{0}=5$. For the unsplit soliton, the variational profile is included too.

$w=\sqrt{2 \pi} /\left[N\left(g_{12}-g\right)\right]$, and a prediction for $N$ at the splitting point,

$$
N_{\text {split }}^{4}=\frac{8 \pi^{2} V_{0}}{g_{12}\left(g_{12}-g\right)^{3}} \exp \left[-\frac{2 \pi}{\left(g_{12}-g\right)^{2}}\right] .
$$

\section{NUMERICAL RESULTS}

Symmetric solitons. Equation (1) was discretized using the Crank-Nicholson scheme and solved numerically in real time, until the solution would converge to a stationary soliton. This way of generating the solitons guarantees their stability. In Fig. 1, we present typical profiles of split and unsplit symmetric solitons, with $N_{1}=N_{2}$. Due to the symmetry, these solitons are always of the intragap type (in Fig. 1, they belong to the second band gap; in the semi-infinite and first gaps, the shape of the solitons is quite similar).

In all cases, the difference between the variational and numerical shapes of the unsplit solitons is extremely small. The present solitons are essentially confined to a single cell of the OL potential. They change the shape and develop undulating tails, which are often considered as a characteristic feature of GSs, when $\mu$ is taken very close to an edge of the band gap (Fig. 1 demonstrates that, even in a wellpronounced split state, peaks of both components stay in a common cell). It is also observed that, as might be expected, the increase of the intraspecies nonlinearity coefficient $g$ pushes the solitons to higher band gaps, while the increase of $g_{12}$ tends to split the two components of the soliton. In addi-

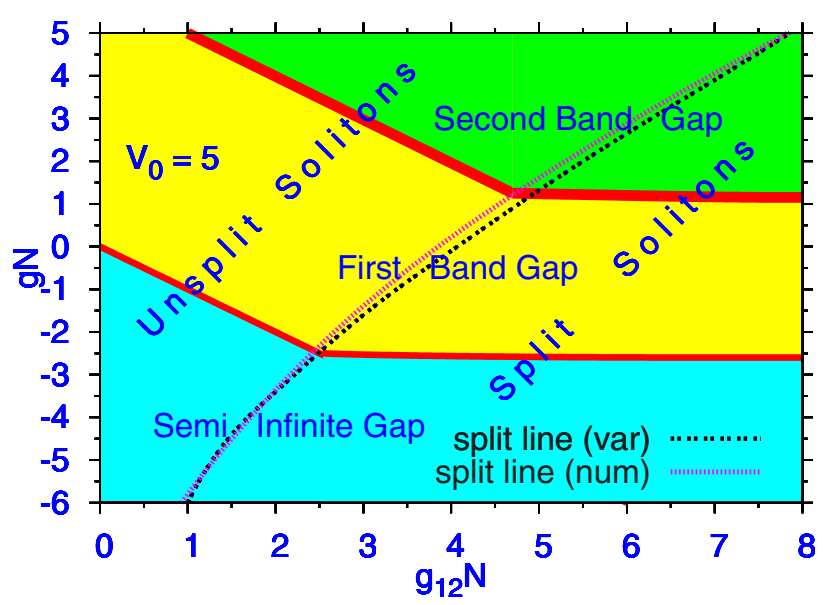

FIG. 2. (Color online) The family of the two-component symmetric solitons $\left(N_{1}=N_{2} \equiv N\right)$, mapped into the plane of the interaction strengths $g_{12} N$ and $g N$. The plane is divided into regions corresponding to the semi-infinite gap and the finite first and second band gaps. They are separated by narrow stripes representing the Bloch bands. The border between the unsplit and split solitons is shown as found from the numerical data, and as predicted by Eq. (11).

tion to the compact GSs presented here, there may also exist loosely bound ones, that extend over several OL [15].

In Fig. 2, the entire family of the symmetric solitons is displayed in the parameter plane of the strength of the intraspecies and interspecies interactions $\left(g N, g_{12} N\right)$. The VA prediction for border between the unsplit and split solitons, given by Eq. (11), provides a remarkably accurate fit to the numerical findings.

Dependences $N(\mu)$ for families of symmetric solitons are plotted in Fig. 3. It is known that a necessary stability condition for solitons populating the semi-infinite gap is given by the Vakhitov-Kolokolov (VK) criterion, $d N / d \mu<0$ [16],

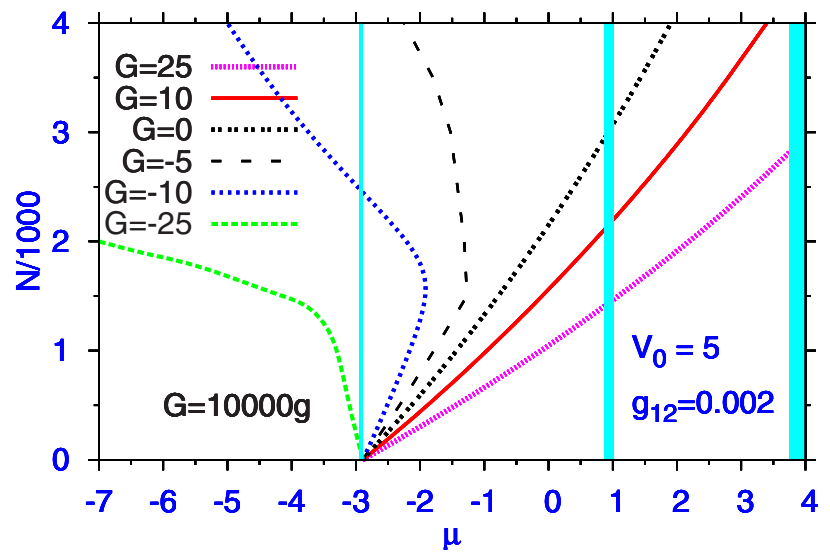

FIG. 3. (Color online) The number of atoms in the symmetric soliton, $N_{1}=N_{2} \equiv N$, versus the common chemical potential of both components, at several values of $g$ for $g_{12}=0.002$. Vertical stripes are the Bloch bands between the gaps (the solution branch with $g$ $=-0.001$ suffers a discontinuity when it hits the band separating the semi-infinite and first finite gaps). 

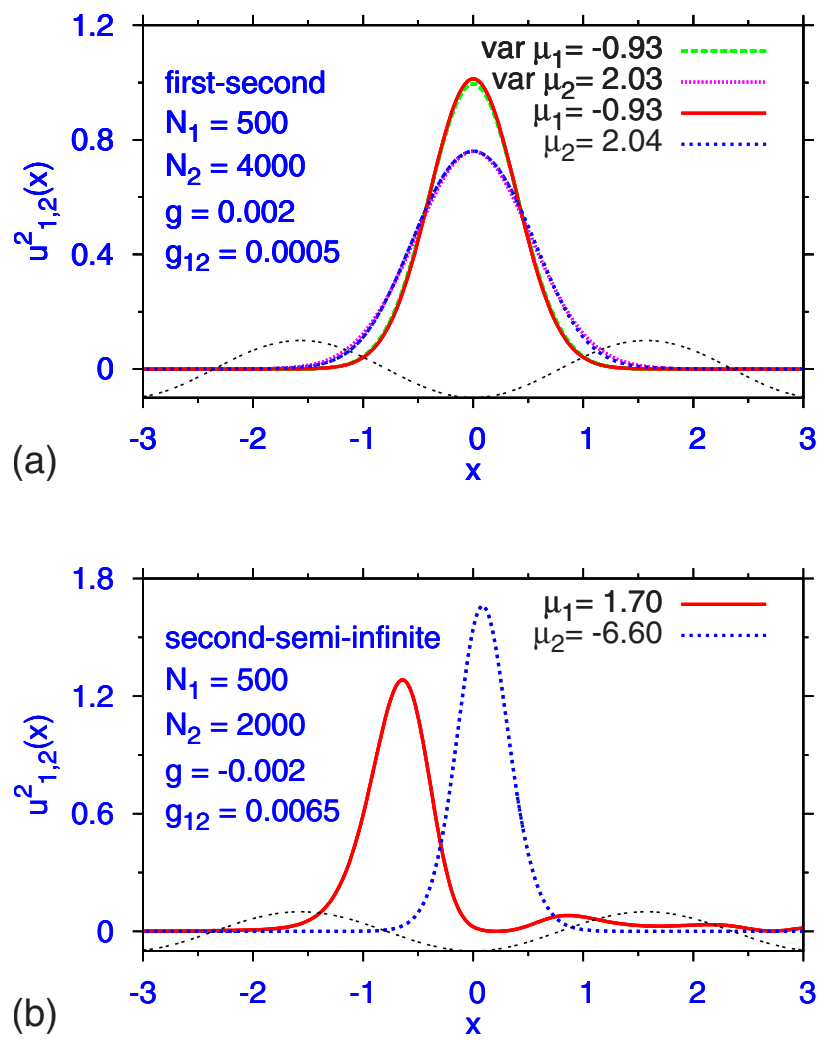

FIG. 4. (Color online) Typical profiles of unsplit and split asymmetric $\left(N_{1} \neq N_{2}\right)$ solitons. The examples represent solitons of intergap types, as indicated in the panels. For the unsplit soliton, the profiles predicted by the VA are shown too.

while stable solitons in finite band gaps have $d N / d \mu>0$, disobeying this criterion $[3,15]$. In the present case, Fig. 3 shows the same generic feature (the semi-infinite gap contains solitons only for $g<0$, i.e., in the case of the selfattraction). A noteworthy feature, viz, a turning point in dependence $N(\mu)$, is exhibited for $g=-0.001$ by the solution branch which passes from the semi-infinite gap into the first finite band gap, and also by the branch corresponding to $g$ $=-0.0005$. Consequently, two different stable solitons can be found in the corresponding interval of $\mu$. The solitons belonging to the branches with $g=0.0025, g=0.001$, and $g=0$ in Fig. 3 are unsplit, and they are accurately predicted by the VA. Accordingly, the curves for these branches, as obtained from the VA and from the numerical data, are virtually identical. On the other hand, all solitons belonging to the branch with $g=-0.0025$ exhibit splitting. As concerns the bending branches, their parts below the turning point are formed by unsplit solitons (which are accurately approximated by the VA), while above the turning point the family continues in the split form. Accordingly, the turning point on each bending branch belongs to the splitting border for the symmetric solitons, cf. Fig. 2.

Asymmetric solitons. Typical examples of solitons with $N_{1} \neq N_{2}$ are displayed in Fig. 4. Similar to their symmetric counterparts, cf. Fig. 1, they feature both unsplit and split shapes (the former ones are well approximated by the VA), which are again confined to a single cell of the OL potential.
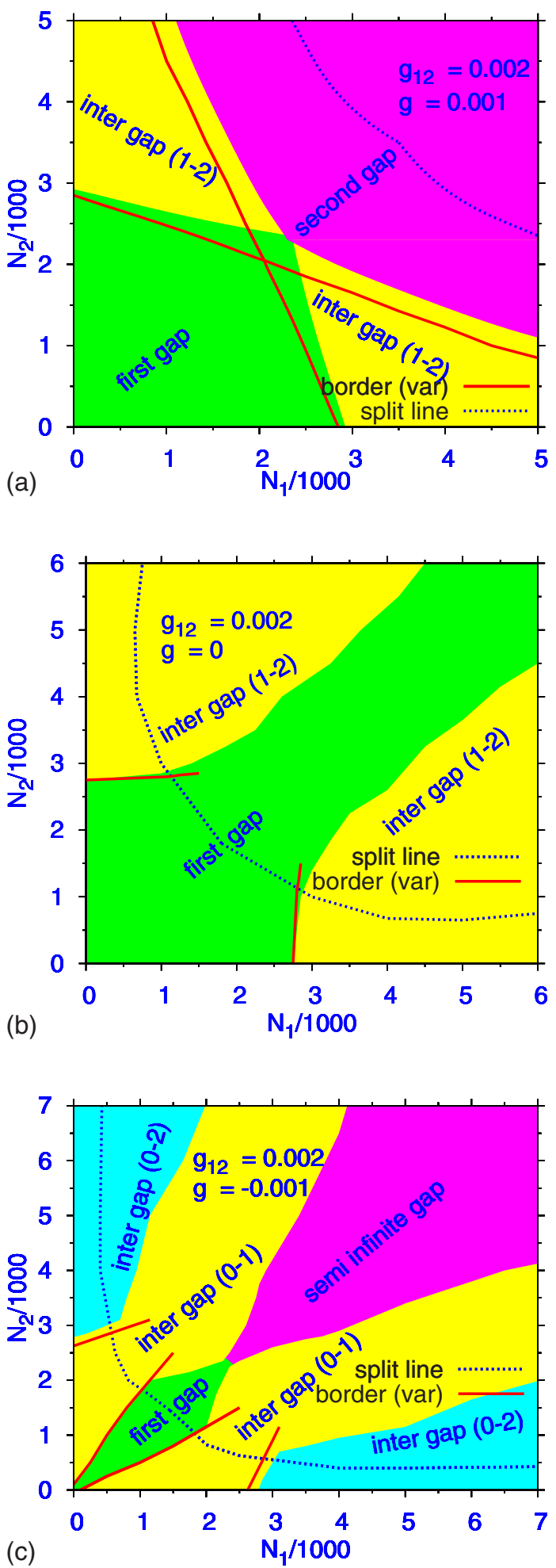

FIG. 5. (Color online) Families of asymmetric and symmetric solitons mapped into the plane of atom numbers $N_{1}$ and $N_{2}$, for different $g_{12}$ and $g$. The plane is divided into regions populated by solitons of six different types (three intragap and three intergap varieties, symbols 0 and 1, 2 standing for the semi-infinite and two lowest finite band gaps, respectively). Each panel also shows the numerically found border between the unsplit and split solitons, and borders between different types of the unsplit ones, as predicted by the VA. 
The entire family of asymmetric and symmetric GSs is mapped in the $\left(N_{1}, N_{2}\right)$ plane, at fixed values of the interaction coefficients $\left(g_{12}\right.$ and $g$ ) in Fig. 5. In these diagrams, the border between intragap solitons of different types shrink to a point belonging to the diagonal line $\left(N_{1}=N_{2}\right)$, which corresponds to symmetric solitons that account for direct transitions between different types of intragap solitons. In Fig. 5 the VA for the unsplit solitons accurately predicts borders between their different varieties.

If none of the nonlinearities is attractive [Figs. 5(a) and 5(b)], no chemical potential may fall in the semi-infinite gap. Three types of GSs are possible if both nonlinearities are repulsive [Fig. 5(a)]: intragap ones, in the two finite band gaps, and the intergap species, combining them. If the intraspecies nonlinearity exactly vanishes [Fig. 5(b)], the interspecies repulsion cannot push both components into the second finite band gap, which leaves us with two species: intragap in the first band gap, and the one mixing the two finite band gaps. The interplay of the attractive intraspecies nonlinearity with the interspecies repulsion supports two intragap and two intergap types, as seen in Fig. 5(c). Note that one of them skips the first band gap, binding together components sitting in the semi-infinite and in second finite gaps. A notable feature of the map in Fig. 5(c) is the smooth transition from ordinary solitons, with both components in the semi-infinite gap, to ones of the semigap type.

\section{CONCLUSION}

In this work, we have considered the interplay of the repulsion between two species of bosonic atoms with intraspe- cies repulsion or attraction in a binary BEC mixture loaded into the OL potential. Families of stable solitons found in this setting are classified as symmetric or asymmetric, split or unsplit, and intragap or intergap. Three varieties of intragap solitons, and another three types of intergap ones are identified, if the consideration is limited to the two lowest finite band gaps of the OL-induced spectrum. Varying the atom numbers in the two components $N_{1,2}$, we have plotted maps of various states. Although different intragap and intergap species are separated by Bloch bands, transitions between them are continuous in the $\left(N_{1}, N_{2}\right)$ plane. In particular, a solution branch which connects the solitons (of the split type), populating the semi-infinite gap, and unsplit solitons in the first finite band gap, features the turning point at the border between the two varieties. Other varieties revealed by the analysis represent semigap solitons, with one component belonging to the semi-infinite gap, and the other one falling into a finite band gap.

A considerable part of the numerical findings reported in this work was accurately predicted by variational approximation. These include the shape of unsplit solitons (both symmetric and asymmetric ones), borders between their varieties, and the splitting border for the symmetric solitons.

\section{ACKNOWLEDGMENTS}

We appreciate support from FAPESP and CNPq (Brazil), and Israel Science Foundation (Center of Excellence Grant No. 8006/03).
[1] V. M. Pérez-García, H. Michinel, and H. Herrero, Phys. Rev. A 57, 3837 (1998); F. Kh. Abdullaev et al., Int. J. Mod. Phys. B 19, 3415 (2005).

[2] K. E. Strecker et al., Nature (London) 417, 150 (2002); L. Khaykovich et al., Science 256, 1290 (2002); S. L. Cornish, S. T. Thompson, and C. E. Wieman, Phys. Rev. Lett. 96, 170401 (2006).

[3] O. Zobay, S. Potting, P. Meystre, and E. M. Wright, Phys. Rev. A 59, 643 (1999); A. Trombettoni and A. Smerzi, Phys. Rev. Lett. 86, 2353 (2001); B. B. Baizakov, V. V. Konotop, and M. Salerno, J. Phys. B 35, 51015 (2002); P. J. Y. Louis, E. A. Ostrovskaya, C. M. Savage, and Y. S. Kivshar, Phys. Rev. A 67, 013602 (2003).

[4] K. M. Hilligsøe, M. K. Oberthaler, and K.-P. Marzlin, Phys. Rev. A 66, 063605 (2002); D. E. Pelinovsky, A. A. Sukhorukov, and Y. S. Kivshar, Phys. Rev. E 70, 036618 (2004).

[5] B. Eiermann, T. Anker, M. Albiez, M. Taglieber, P. Treutlein, K. P. Marzlin, and M. K. Oberthaler, Phys. Rev. Lett. 92, 230401 (2004); O. Morsch and M. Oberthaler, Rev. Mod. Phys. 78, 179 (2006).

[6] V. Ahufinger, A. Sanpera, P. Pedri, L. Santos, and M. Lewenstein, Phys. Rev. A 69, 053604 (2004).

[7] M. Matuszewski, W. Krolikowski, M. Trippenbach, and Y. S. Kivshar, Phys. Rev. A 73, 063621 (2006).
[8] C. J. Myatt, E. A. Burt, R. W. Ghrist, E. A. Cornell, and C. E. Wieman, Phys. Rev. Lett. 78, 586 (1997); D. M. StamperKurn, M. R. Andrews, A. P. Chikkatur, S. Inouye, H. J. Miesner, J. Stenger, and W. Ketterle, ibid. 80, 2027 (1998).

[9] A. Simoni, F. Ferlaino, G. Roati, G. Modugno, and M. Inguscio, Phys. Rev. Lett. 90, 163202 (2003).

[10] V. M. Pérez-García and J. B. Beitia, Phys. Rev. A 72, 033620 (2005); S. K. Adhikari, Phys. Lett. A 346, 179 (2005); Phys. Rev. A 72, 053608 (2005); J. Phys. A 40, 2673 (2007).

[11] A. Gubeskys, B. A. Malomed, and I. M. Merhasin, Phys. Rev. A 73, 023607 (2006).

[12] M. Matuszewski, B. A. Malomed, and M. Trippenbach, Phys. Rev. A 76, 043826 (2007).

[13] V. M. Pérez-García, H. Michinel, J. I. Cirac, M. Lewenstein, and P. Zoller, Phys. Rev. A 56, 1424 (1997).

[14] L. Salasnich, A. Parola, and L. Reatto, Phys. Rev. A 65, 043614 (2002); L. Salasnich and B. A. Malomed, ibid. 74, 053610 (2006).

[15] S. K. Adhikari and B. A. Malomed, Europhys. Lett. 79, 50003 (2007); Phys. Rev. A 76, 043626 (2007).

[16] M. G. Vakhitov and A. A. Kolokolov, Izv. Vuz. Radiofiz. 16, 1020 (1973) [Sov. J. Radiophys. Quantum Electr. 16, 783 (1973)]. 